\section{Immune reaction to food antigens in Graves'disease (GD) patients: role of gliadin and other food antigens}

Danila Covelli ${ }^{1}$, Giuseppe Colucci ${ }^{1}$, Mario Salvi ${ }^{1}$, Ulrike Kaiser $^{2}$, Anja Eckstein ${ }^{2}$, Maria Cristina Burlacu ${ }^{3}$, Chantal Daumerie ${ }^{3}$, Gez Richell ${ }^{4}$, Petros Perros ${ }^{4}$, Mohd $^{2}$ Shazli Draman ${ }^{5}$, Marian Ludgate ${ }^{5}$, Giulia Masetti ${ }^{6}$ \& Filippo Biscarini ${ }^{6}$

\section{Author affiliations}

${ }^{1}$ Graves' Orbitopathy Centre, Endocrinology, Fondazione IRCCS Cà Granda, and University of Milan, Milan, Italy; ${ }^{2}$ Center for Ophthalmology University Clinic, Essen, Germany; ${ }^{3}$ Médecin spécialiste hospitalier Service d'Endocrinologie, Diabétologie et Nutrition Cliniques universitaires St-Luc (UCL), Bruxelles, Belgium; ${ }^{4}$ Diabetes \& Endocrine Royal Victoria Infirmary, Newcastle Upon Tyne, UK; ${ }^{5}$ Division of Infection and Immunity School of Medicine, Cardiff University, Cardiff, UK; ${ }^{6}$ PTP Science Park, Lodi, Italy.

As known, an imbalance of the gut microbiota is associated with a higher risk for autoimmune diseases. Moreover the increased rates of autoimmunity reported in urban residential areas worldwide suggest a possible influence of diet. We report on the antibody response to food antigens in GD patients. Since $10 \%$ of celiac patients develop an autoimmune thyreopathy we focused on gliadin (DGP), transglutaminases (tTG) and 40 other food antigens (FA). Commercially available ELISA assays were performed according to the manufacturer's instructions. 105 and 108 sera from 5 European endocrine centres have been tested for IgG and IgA to TTG and DGP. Results have been compared to epidemiological data. 71 sera have also been tested for IgG to FA and compared to 25 healthy controls. 6 out of 105 sera (5.7\%) showed positive tTG; 16 and 7 out of 108 (15 and 6.5\%) positive DGP-IgA and IgG, respectively; a higher prevalence compared to the worldwide prevalence of celiac disease $(1 \%)$ (chi-squared test; $p$-value $<0.001$ ).

Prevalence of smokers and ocular involvement was not higher in patients with positive sera compared to negative. 23 out of $71(32.3 \%)$ GD sera showed sensitivity against a food antigens, compared to $25 \%$ ( 6 out of 24 ) positive results among healthy controls (chisquared test; P-value=0.4). Interestingly, some antigens (cow's milk, egg white, wheat, yeast) are more frequently positive than others. The distribution of antibodies against TSH receptor (TRAb) values was not different in positive or negative sera. In conclusion, the prevalence of positive tTG antibodies is higher in GD patients than worldwide. Even though autoantibodies to DGP and tTG were equally distributed between all 5 centres we observed the highest percentage of positive responses to other food antigens in Cardiff, suggesting that diet may contribute to the increased sensitivity. More studies are needed to confirm these data.
ECE 2017

19th European Congress of Endocrinology

19th European

Congress of Endocrinology

Lisbon, Portugal

20 May 2017 - 23 May 2017

European Society of Endocrinology

Other volumes from this event series: European Congress of

Endocrinology 
